\title{
Light shaping from a physical-optics point of view
}

\author{
Liangxin Yang ${ }^{1, *}$, Irfan Badar $^{1,2}$, Christian Hellmann ${ }^{2,3}$, and Frank Wyrowski ${ }^{1}$ \\ ${ }^{1}$ Institute of Applied Physics, Friedrich Schiller University Jena, Jena, Germany \\ ${ }^{2}$ Wyrowski Photonics UG, Jena, Germany \\ ${ }^{3}$ LightTrans International UG, Jena, Germany
}

\begin{abstract}
In the design of optical element for light shaping, a geometric-optics assumption is usually used, where the validity of the assumption is rarely discussed in literature. In this work, the field tracing techniques for modeling light-shaping systems are presented, which reveals the optical element resulted from those geometric-base algorithm is not always accurate enough for the design task. An example is demonstrated with the functional embodiment of the element. The simulation result shows that diffraction effect may occur, especially in paraxial situation. However, the designed result start with the assumption is well-introduced initial guess for further optimization with the iterative Fourier transform algorithm (IFTA).
\end{abstract}

\section{Introduction}

The design of a light-shaping system is aimed at redistributing the energy of the light source for certain applications. In literature, different algorithms have been proposed, attempt to find a suitable optical element to fulfill the task. One type of algorithms is based on the geometric assumption, where a one-to-one map between the source energy distribution and the target one is assumed, and a local relationship between their coordinate is established. By using the assumption, either a phase-only holography optical element(HOE) [1, 2], or a freeform surface can be designed $[3,4]$.

Typically, for analyzing the design result, a ray-tracing simulation is performed, which gives a result corresponding exactly to the target, and is usually given as proof of the validity of the design. However, using a simulation technique which is also under the geometric-optics assumption is not enough for a full analysis of the accuracy of the design, since any eventual physical-optics effects would go unnoticed, both in the design and in the subsequent simulation. Therefore, to answer the question of whether the geometric-optics assumption is accurate enough for the design algorithm, the designed result has to be investigated via physical-optics simulation.

\section{Field tracing techniques}

In this work, we present a field tracing technique under physical optics for modeling the light-shaping system. The modeling methods are sequentially applied in the light path and illustrated in a field tracing diagram as shown in Fig. 1. The field tracing diagram builds up an algorithm demonstrates how the target field is calculated from the

*e-mail: liangxin.yang@uni-jena.de input,

$$
\left.\boldsymbol{E}^{\text {out }}\left(\boldsymbol{\rho}^{\prime}\right)\right|_{\text {par }}=\mathcal{F}^{-1}\left\{\tilde{\mathcal{P}} \mathcal{F}\left\{\left.\mathcal{B} \boldsymbol{E}^{\text {in }}(\boldsymbol{\rho})\right|_{\mathrm{pin}}\right\}\right\} .
$$

where all the $\boldsymbol{E}$ are the vectorial electric fields, defined either in the space domain, with $\rho=(x, y)$ or the spatialfrequency domain, with $\boldsymbol{\kappa}=\left(k_{x}, k_{y}\right) . \mathcal{B}$ is an operator in general, which acts on the field in the $\rho$ domain. $\tilde{\mathcal{P}}$ denotes the free space propagation operator in the $\kappa$ domain. And $\mathcal{F}$ and $\mathcal{F}^{-1}$ are the direct and inverse Fourier transform operators for the fields between two domains.

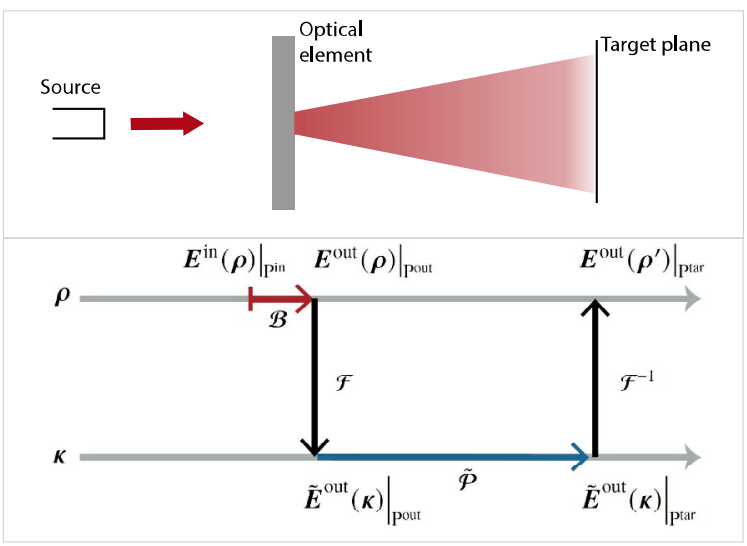

Figure 1. A light-shaping system and its field tracing diagram.

Numerically, there are several algorithms available to compute the Fourier transform in Eq. 1 [5, 6], e.g. Fast Fourier Transform (FFT), semi-analytical Fourier Transform (SFT), or Homeomorphic Fourier Transform (HFT). HFT and inverse HFT are approximated pointwise Fourier transforms, which produce a one-to-one map between the coordinates of the fields expressed in the $\rho$ domain and in the $\kappa$ domain [6]. 
In the field tracing diagram, $\tilde{\mathcal{P}}$ is a pointwise operator. Therefore, if the $\mathcal{B}$ is also a pointwise operator, and all the direct Fourier transforms in the field tracing can be approximated by the HFT, the whole field tracing simulation of the light-shaping system gives a pointwise calculation, which forms the homeomorphism between $\left.\boldsymbol{E}^{\text {in }}(\boldsymbol{\rho})\right|_{\text {Pin }}$ and $\left.\boldsymbol{E}^{\text {out }}\left(\boldsymbol{\rho}^{\prime}\right)\right|_{\mathrm{ptar}}$, i.e. $\boldsymbol{\rho}^{\prime}(\boldsymbol{\rho})$ is a bijective mapping function. And that is the typical assumption usually made in freeform surface design algorithms. However, in certain design task, the assumption is not always satisfied, especially the accuracy of the HFT is not always sufficient.
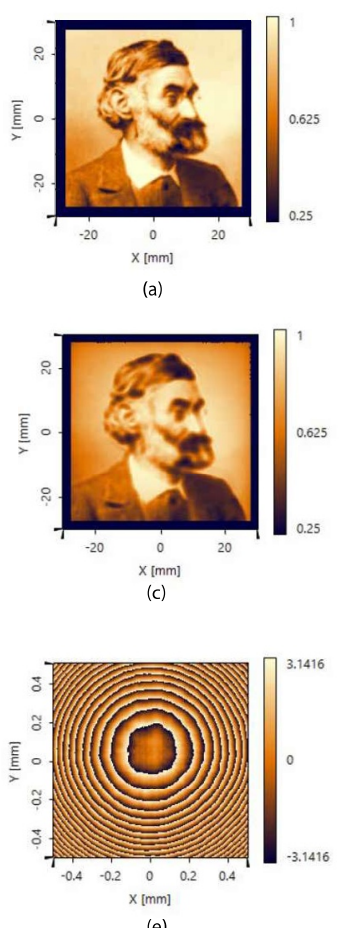
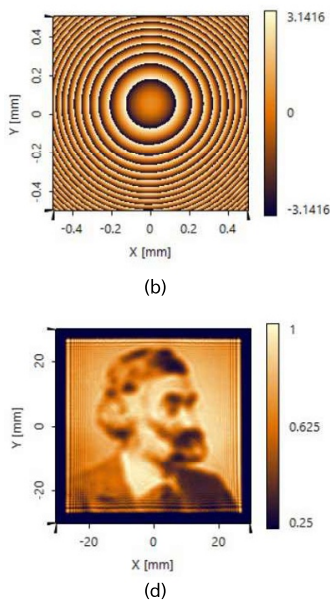

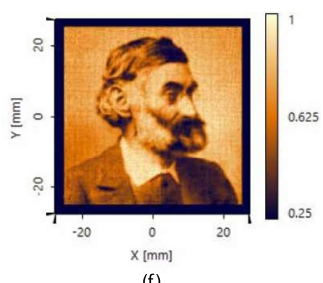

Figure 2. (a) Target irradiance distribution (normalized). (b)The wrapped phase response function designed with geometric assumption (Unit: rad). (c) Irradiance: simulation result, with the HFT used. (d) Irradiance: simulation result, with the FFT used. (e)The optimized phase response function by IFTA (Unit: rad). (f) Irradiance: simulation result with the optimized phase response function

\section{Example and discussion}

For investigating the assumption in the design algorithm, an example is illustrated in Fig. 2 to demonstrate the effect. A target irradiance pattern is shown in Fig. 2 (a). The design task is to shape an input plane wave for achieving the target irradiance distribution in the far field zone. The input beam size is $1 \mathrm{~mm}$ and the target plane is set perpendicular to the optical axis and located $1 \mathrm{~m}$ away from the component.

The functional embodiment of the optical element, which is considered as a phase response function, is designed by using the geometric-optics assumption where a

local mapping between the irradiance of the input and the target is established. The mathematical model of "optimal mass transport (OMT)" [7] is applied to calculate the mapping. When the mapping is obtained, the phase response function can be concluded from geometric relationship [2]. The designed result is shown in Fig. 2 (b).

With the phase response function, simulation is performed by the field tracing techniques. $\mathcal{F}$ and $\mathcal{F}^{-1}$ are selected as HFT to start with. Therefore, the assumption in the design algorithm is also used in the simulation. The irradiance at the target plane is measured in the detector, and shown in Fig. 2 (c). For further investigation, the $\mathcal{F}$ and $\mathcal{F}^{-1}$ are switched to rigorous FFT. The result is shown in Fig. 2 (d). The result in Fig. 2 (c) coincides with the target in Fig. 2 (a), however, the result in Fig. 2 (d) shows obvious diffraction effect and low resolution, which proves that in this system, the HFT is not accurate enough for the calculation of the Fourier transform, so that the one-to-one map between the input and target does not exist, i.e. the design from the algorithm with geometric-optics assumption is not valid.

For optimization of the result, the iterative Fourier transform algorithm (IFTA) is performed, starting with an initial guess given by previous result. The optimized phase response function is shown in Fig. 2 (e). Comparing the results, one can see that the phase response function after IFTA is quite closed to the initial one, so it is reasonable to conclude the result from the mapping type algorithm as a good option to start with. Fig 2 (f) shows the simulation result with the optimized function, which compared to the irradiance distribution in Fig. 2 (d), the diffraction fringe has been removed and the resolution is improved.

All the simulation are done via the software VirtualLab Fusion [8].

\section{References}

[1] H. Aagedal, M. Schmid, S. Egner, J. Müller-Quade, T. Beth, F. Wyrowski, J. Opt. Soc. Am. A 14, 1549 (1997)

[2] A. Hermerschmidt, H.J. Eichler, S. Teiwes, J. Schwartz, Proc. SPIE 3291, 40 (1998)

[3] Z. Feng, L. Huang, G. Jin, M. Gong, Opt. Express 21, 28693 (2013)

[4] R. Wu, Z. Feng, Z. Zheng, R. Liang, P. Benítez, J.C. Miñano, F. Duerr, Laser \& Photonics Reviews 12, 1700310 (2018)

[5] Z. Wang, S. Zhang, O. Baladron-Zorita, C. Hellmann, F. Wyrowski, Opt. Express 27, 15335 (2019)

[6] Z. Wang, O. Baladron-Zorita, C. Hellmann, F. Wyrowski, Opt. Express 28, 10552 (2020)

[7] C. Prins, R. Beltman, J. ten Thije Boonkkamp, W. IJzerman, T. Tukker, SIAM Journal on Scientific Computing 37, B937 (2015)

[8] Physical optics simulation and design software "Wyrowski VirtualLab Fusion", developed by Wyrowski Photonics GmbH, distributed by LightTrans International UG, Jena, Germany. 